\title{
Development of an improved method for identifying material stress-strain curve using repeated micro-impact testing
}

\author{
H. Al Baida ${ }^{a}$, G. Kermouche ${ }^{\mathrm{b}}$, C. Langlade ${ }^{\mathrm{a}, *}$ \\ a Université Technologique de Belfort-Montbéliard, IRTES-LERMPS EA 7274, France \\ ${ }^{\mathrm{b}}$ Ecole des Mines de Saint-Etienne, Centre SMS, LGF UMR 5307 CNRS, France
}

Impact-based Mechanical Surface Treatments such as shot peening are widely used in aerospace, nuclear and other industries to improve the mechanical resistance of components. Measuring the stress-strain curve of materials under high-strain rate using repeated impacts is a key issue to improve such processes. This study presents an exten-sion of a method developed by Kermouche (2013) for identifying the material stress-strain curve. It combines numerical and experimental approach using micro-impact testing. The main originality of the present work is the use of the impact load values instead of the depth of the residual imprint as an input parameter of the inverse identification. The reliability of the proposed method is then checked from a set of numerical blind tests. A direct method derived from Tabor's pioneering work (Tabor, 2000) is also proposed to convert the impact measurements into an approximate stress-strain curve. These two methods have been applied on a commercially pure copper and show very good agree-ment. The main advantage of this analysis is to determine the mechanical behaviour of metallic surface at high strain rate using limited numbers of samples and tests.

\section{Introduction}

Shot peening is a surface treatment process used in many industrial branches to improve the mechanical properties of materials by producing a compressive residual stress from the projection of spherical balls at high speed (between $100-500 \mathrm{~mm} \cdot \mathrm{s}^{-1}$ ). The ball impacts result in large plastic deformations on the treated area that induce surface hardening and beneficial compressive residual stress (Abramov et al., 1998). To further develop this process or apply it to new alloys, a better knowledge of the behaviour of materials under similar process conditions is required. In particular, the determination of the stressstrain curve at high strain rate is of main interest for prediction of strain and residual stress fields.

\footnotetext{
* Corresponding author.
}

Several techniques are available to determine the mechanical behaviour of materials subject to high speed loadings. Among them Hopkinson bar testing is the most widely used (Jaspers and Dautzenberg, 2002). However, it requires specific samples that are material and cost consuming. Moreover the resulting stress-strain curve corresponds to bulk behaviour and does not take into account the effect of surface preparations or surface treatments. It therefore cannot be used to accurately describe the shot-peening process for example. For these reasons, other kinds of mechanical testing have been developed. Some works (Beghini et al., 2006; Collin et al., 2009, 2008) deal with the use of instrumented indentation tests under single or repeated load cycles for determining the stress-strain curve of material. However shot peening consists of a dynamic high speed impact which cannot be repeated in the case of quasi-static methods such as static indentation methods. Other works (Subhash et al., 1999; 
Sundararajan and Tirupataiah, 2006; Tirupataiah and Sundararajan, 1991) are based on the use of specific dynamic indentations performed on instrumented nanoimpact tests. More specifically Lu and al. (Lu et al., 2003) studied the load-depth response of dynamic indentation to determine the strain-rate sensitivity of metals. However it's proved to be costly and challenging especially when the penetration depth is required, which limits their practical use.

In our previous works (Kermouche et al., 2013; Lamri et al., 2010; Sekkal et al., 2005), an experimental setup was designed, based on a commercial micro-marking device (CN312C Technifor ${ }^{\circ}$ ), that projects a spherical indenter with controlled displacement. Combining measurements of radius and depth of the residual scar as a function of the impact number and a database containing the results of numerical simulations, it was possible to extract the stress-strain curve of the impacted material for a strain rate in the range of [100-1000] s ${ }^{-1}$.

However, the determination of the depth of the residual imprint requires 3D measurements which could be timeconsuming and/or inaccurate. This paper presents a new inverse identification which uses the impact load values instead of the depth values. The reliability of the proposed method is then checked from a set of numerical blind tests.

Then, the method is applied on commercially pure copper. The obtained results are then compared to a direct identification based on Tabor pioneering work (Tabor, 2000) and on the model proposed by Kermouche et al for sharp indentation (Kermouche et al., 2008), the latter being based on a direct analysis of the impact tests.

\section{The repeated impact set-up}

The repeated impact device Fig. 1 has been extensively described in previous papers (Kermouche et al., 2013; Lamri et al., 2013). Thus only the main features are described in this paper. An electromagnetic system pushes a rigid indenter (spherical end) into the sample surface at high speed and normal incidence. The power control of the electromagnet as well as the initial distance between

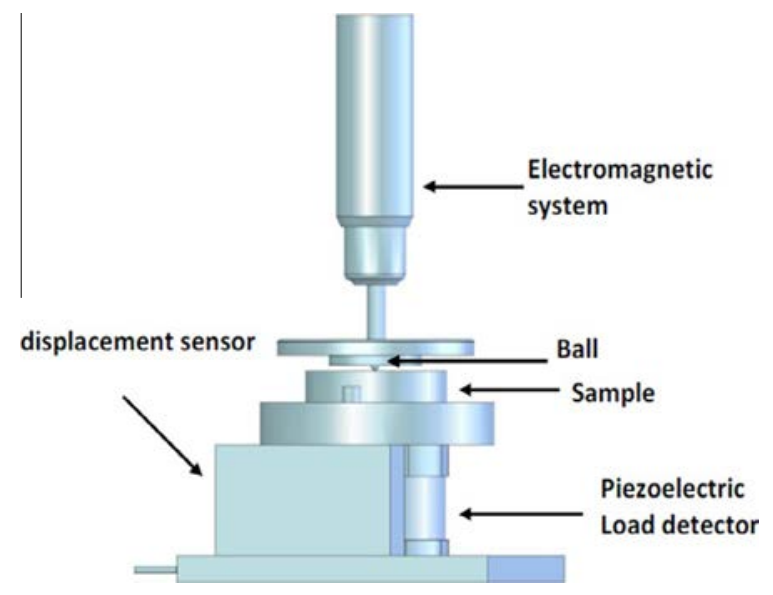

Fig. 1. Instrumented impact testing device. the indentor and the samples allow to settle the impact energy. During this work, $2 \mathrm{~mm}$ diameter Zirconia balls (grade 10) ( $E=200 \mathrm{GPa}$, hardness: $800 \mathrm{Hv}$ ) have been used as an impacting tip, leading to a total indenter mass of $169.9 \mathrm{~g}$.

As the impact frequency (controlled by electromagnets) is kept constant at $10 \mathrm{~Hz}$ and impact durations are in the $10 \mathrm{sec}$ range, ultra-fast displacement sensor (EOTECH SA) tracks the movement of the ball before, during and after the impact, then the indenter velocity can be deduced from the displacement of the indenter. Considering the indenter weight, the kinetic energy can be determined by $E_{\text {impact }}=m v^{2} / 2$, where $m$ the indenter mass and $v$ the indenter speed when it hits the sample surface. During each impact, the normal component of the induced load is recorded using a piezoelectric load sensor (KISTLER). The impact load ranges from 50 to $2500 \mathrm{~N}$. The technical characteristics of the two sensors were specifically chosen to be relevant with the impact conditions (impact duration, repetition frequency, measuring range...).

The usual impact energy ranges from [1 to 21] mJ, which corresponds to an impact speed of $100-500 \mathrm{~mm} / \mathrm{s}$ and thus to a representative material strain rate equivalent to $[100-1000] \mathrm{s}^{-1}$. The letter can be computed as the ratio between the normal impact speed and the contact radius. This representative material strain and its definition that can appear quite simplistic is frequently used in indentation studies as the strain rate field is not homogenous and does not depend on time (Kermouche et al., 2013).

\section{Finite element model}

\subsection{Presentation}

Repeated impacts have been modelled using non-linear dynamic axisymmetric simulations in Abaqus Explicit (Systems, 2011). The mesh has been created using linear elements with different grades. It has been specially refined near the contact zone (Fig. 2) in order to get more accurate radius and load values.

The tested substrate is an elastic-plastic solid considered as semi-infinite with isotropic hardening work. It

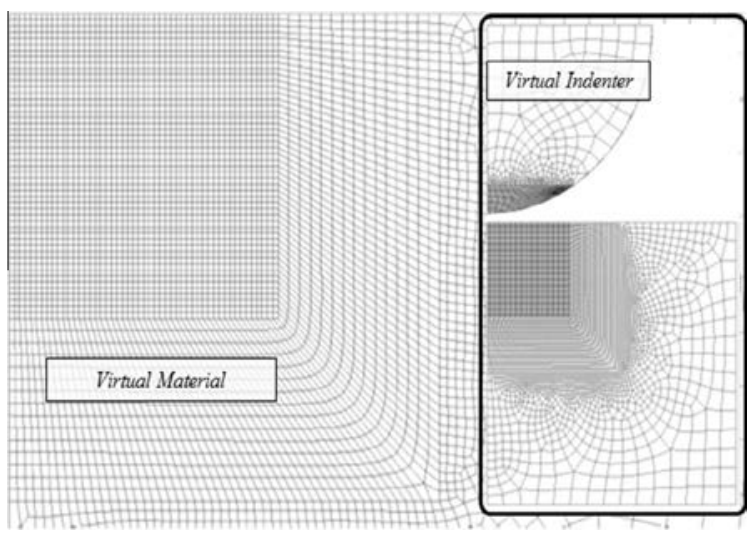

Fig. 2. Finite element model. 
can be noted that, in the case of spherical indentation, it is generally assumed that the hardening effects of the material are governed by pure isotropic hardening (Huber and Tsakmakis, 1999). Moreover, most authors are using isotropic hardening conditions in the modelling of shot peening process (Miao et al., 2011) and staticindentation process (Collin et al., 2010).

The ball, used as indentor, is a deformable elastic solid with a radius of $1 \mathrm{~mm}$. Its displacement depends on its initial speed and the contact is assumed to be frictionless. Previous studies (Kermouche et al., 2013) have already shown that the friction coefficient value does not significantly change the radius and load values using our standard experimental conditions. Previous experimental and numerical works have also shown that the main impact scar modifications occur during the first 10 cycles. After 10 impacts, radius and load values remain almost constant until the occurrence of the wear which is generally generated after few hundreds of impacts.

In addition, a comparison was performed by Kermouche et al. (2013) between a quasi-static impact model created under Systus software and a dynamic impact model using the Abaqus software. Starting with the same impact parameters, the two models give the same results of impact residual radius and depth. Based on this result, it can be deduced that in the considered impact speed range, the dynamic effect can be neglected. As shown by Johnson (1985) a quasi-static approach can be used for modelling the dynamic impact if the impact time is higher than the elastic wave propagation time in the contact area. This condition can be written as

$t_{\text {impact }} \geqslant \frac{2 a}{c}$

Where $t_{\text {impact }}$ is the impact time, $a$ the contact radius and $c$ the longitudinal elastic wave. In this study, the contact radius is around $0.5 \mathrm{~mm}$, the wave propagation speed in a steel is approximately $5 \times 10^{6} \mathrm{~mm} / \mathrm{s}$. This leads to a ratio of $2 a / c$ of about 200 ns well shorter than the impact time $t_{\text {impact }}$ of about $200 \mu \mathrm{s}$.

The question of eventual thermal effects has also to be addressed. Previous study (Kermouche et al., 2013) shows that after 20 repeated impacts on steel, simulated using the Johnson-Cook law and the proportion of plastic work dissipated under thermal form is taken to be about $90 \%$, the temperature rise is not greater than $80^{\circ} \mathrm{C}$. No thermal effect will therefore be taken into account in the present work.

\subsection{Validation}

To validate the experimental load measurement system as well as the FEM model, tests on treated M2 sample (AISI M2 high speed, quenched and thermally treated to achieve 62-64 HRC) have been performed under purely elastic conditions. Fig. 3 shows the discrepancy between the experimental and simulated signals. The impact speed is $402 \mathrm{~m} . \mathrm{s}^{-1}$, the experimental impact time is $2.8 \mathrm{E}^{-4} \mathrm{~s}$ and the numerical impact time is $2.4 \mathrm{E}^{-4} \mathrm{~s}$.

The detected gap between the maximal load values of the two curves $\left(F_{n u m}=1050 \mathrm{~N}\right.$ and $\left.F_{\text {exp }}=958 \mathrm{~N}\right)$ is around

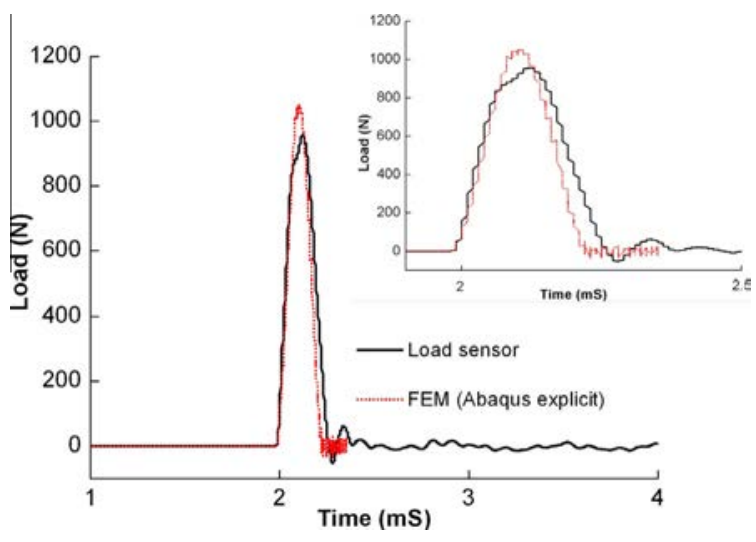

Fig. 3. Comparison between experimental and numerical impact load as a function of time for AISI M2 high speed steel under pure elastic impact conditions.

8.7\%. By comparison, the use of the classical Hertz equation (Johnson, 1985) leads to a theoretical value of $\mathrm{F}=985 \mathrm{~N}$ that is in very good agreement with both experimental and numerical values.

\section{The inverse identification of stress-strain curve}

\subsection{Two-parameter power hardening law}

Various mathematical stress-strain curves may be found in the literature to simulate that of metals. Depending on the number of adjustable parameters, these stress-strain curves may consider complex mechanical effects. Among various possibilities, many authors (Beghini et al., 2006; Dao et al., 2001; Huang et al., 2011; Moussa et al., 2014a,b) proposed to use the Hollomon's equation to describe common behaviour of metals. The Hollomon's equation can be expressed as follows: $\sigma=k \varepsilon^{n}$ (Chollacoop et al., 2003; Dao et al., 2001; Lee et al., 2009) where $\mathrm{k}$ is the strength coefficient and $\mathrm{n}$ the strain hardening exponent. It is worth noting that Hollomon's law, as well as Johnson-Cook's, is a good approximation of most metal behaviour under monotonic loadings. It is also to be noted that Hollomon's law does not consider any strain rate or thermal influence (Kermouche et al., 2013). However, due to the specific testing parameters used in this study, strain rates ranging from [100 to 1000$] \mathrm{s}^{-1}$ and contact temperatures are expected. For most metals, this range is limited enough to assume that an equivalent time-independent stress-strain curve can be used to model the mechanical response of the surface.

\subsection{Parametric study and result}

As the strategy developed in this paper is to determine the best stress-strain curve that allows to obtain the best approach regarding experimental radius of residual imprint and the measured impact load for a given impact energy $E_{\text {impact }}$, an extensive parametric study has thus been first carried out including different $\left[k, n, E_{\text {impact }}\right]$ parameters, in order to cover a large range of possible stress-strain 
curves and impact conditions. Moreover, several databases have been built for different Young modulus values in order to be used for different types of metallic alloys.

The input parameters of the finite element model are:

- $k:[500,1000,2000,3000,4000,5000] \mathrm{MPa}$

- $n:[0.0,0.1,0.2,0.3,0.4,0.5]$

- $E_{\text {impact }}=[1,8,17] \mathrm{mJ}$ for $E=210 \mathrm{GPa}$ and $v=0.3$

Or $E_{\text {impact }}=[3,17] \mathrm{mJ}$ for $E=75 \mathrm{GPa}$ and $v=0.3$

Or $E_{\text {impact }}=[9,17] \mathrm{mJ}$ for $E=105 \mathrm{GPa}$ and $v=0.3$

Or $E_{\text {impact }}=[2,3,14] \mathrm{mJ}$ for $E=180 \mathrm{GPa}$ and $v=0.3$

The ceramic indenter is assumed to be perfectly elastic with $E=210 \mathrm{GPa}$ and $v=0.25$ for Zirconia.

For each finite element simulations, 10 load/unload cycles, corresponding to 10 impacts, were simulated on a virtual material characterised by a $(k, n, E, v)$ set of parameters.

The pure elastic behaviour of indenter indicates that a part of the impact energy is stored as elastic deformation of the indenter. For each numerical simulation corresponding to a given set of $\left[k, n, E_{\text {impact }}\right]$, the changes in load and radius with the number of impacts are obtained as shown in Fig. 4. Referring to the previous work (Kermouche et al., 2013), these variations can be fitted as

$x(N)=A_{x} \ln (N)^{2}+B_{x} \ln (N)+C_{x}$

where $N$ is the number of impacts, $x$ is the characteristic parameter of the impact, i.e. radius $r$ and/or load $f$. This specific fitting function Eq. (2)has also been chosen in accordance with indentation testing studies (Chollacoop et al., 2003; Dao et al., 2001; Huang et al., 2011; Lee et al., 2009). $A_{x}, B_{x}, C_{x}$ are functions of $\left[k, n, E_{\text {impact }}\right]$ and have been stored in two databases: one for the load growth $\left(A_{f}, B_{f}, C_{f}\right)$ and the other for the growth of radius $\left(A_{r}, B_{r}, C_{r}\right)$.

\subsection{Identification process}

Experimental results can also be approximated using Eq. (2). Denote $r e(N)$ and $f e(N)$ the experimental values of

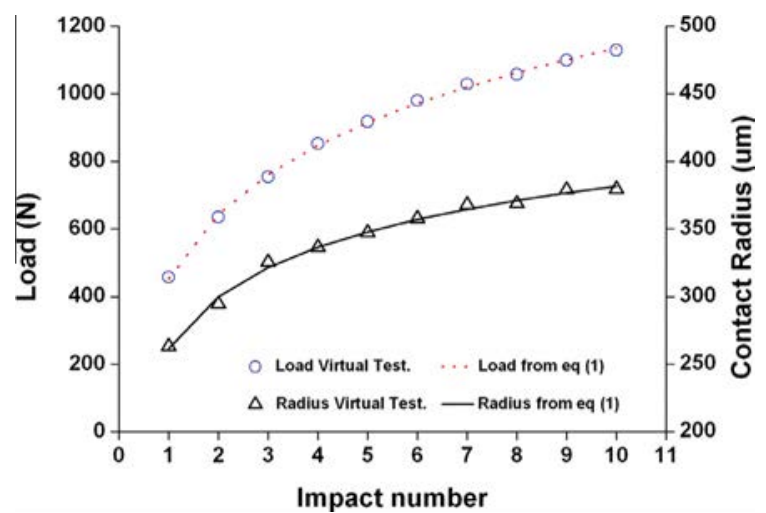

Fig. 4. Evolution of load and residual radius per impact versus number of impacts obtained by numerical simulation $(k=2000 ; n=0.3$ and $E_{\text {impact }}=8 \mathrm{~mJ}$ ) and fitted from Eq. (1). the residual imprint radius and impact load after $N$ impacts at a given energy.

With the experimental curves $r e(N)$ and $f e(N)$ and the numerical curves $r(N)$ and $f(N)$ as a function of $\left[k, n, E_{\text {impact }}\right]$, an inverse identification may be developed to identify the best $\left[k, n, E_{\text {impact }}\right]$ values to reach experimental values.

Considering the impact energy $E_{\text {impact }}$, the optimal values of $k$ and $n$ are determined by fitting the minimum value of the following function (Kermouche et al., 2013):

$I_{x}(k, n)=100 * \frac{\sqrt{\int_{0}^{N \max }\left(x-x_{\exp }\right)^{2} d N}}{\sqrt{\int_{0}^{N \max } x^{2} d N}}$

where $x$ is the characteristic parameter of the impact, i.e. radius $r$ and/or load $f$. This function is drawn from the general concept of variance, or standard deviation (Kermouche et al., 2013). It is an illustration of the classical least square method and calculates the surface between experimental and predicted radius fitted curve (resp load). Seeking the minimum value of the function $I_{r}(k, n)\left(\operatorname{resp} I_{f}(k, n)\right)$ allows to identify the best couple $(k, n)$ which permits to adjust the experimental variation of the radius (resp load) as a function of the number of impacts. If both minima may correspond, in most cases two sets of values are identified thus it may be difficult to determine which one is the best. Therefore, we propose to seek the minimum value of the function $I_{r}(k, n) * I_{f}(k, n)$ which combines the both parameters and gives them the same weight.

A first validation step was then performed considering a virtual AISI316L stainless steel substrate impacted under $14 \mathrm{~mJ}$. Considering a virtual stress-strain curve, the FEM impact simulation leads to the determination of a set of values of radius and load for each simulation of virtual impacts. The 2 sets were then used to run the inverse identification that leads to the couple $k=977$ and $n=0.259$. The comparison between the first virtual values and those obtained via the proposed method is plotted in Fig. 5. It shows a good agreement between the virtual radius and load evolutions and those resulting from the inverse identification $(k, n)$.

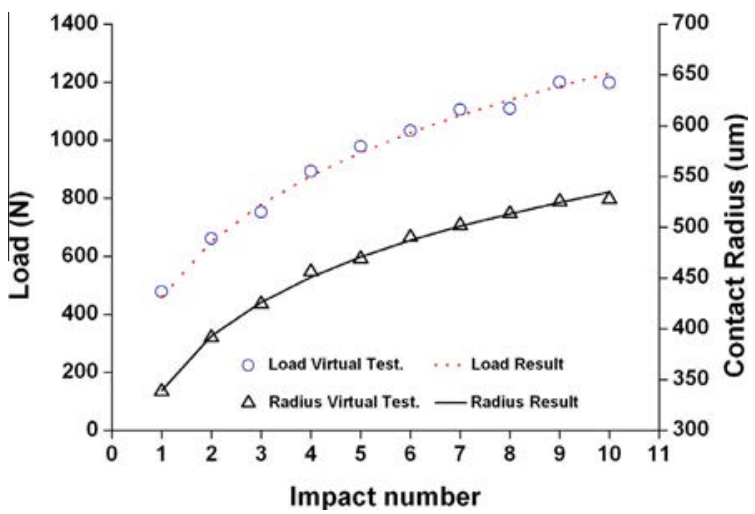

Fig. 5. Load and radius of each impact obtained using the inverse method on result of the numerical virtual test on AISI316L with impact energy of $14 \mathrm{~mJ}$. 


\subsection{Interpolation method}

The previous tests and inverse identifications were performed at a given impact energy of $14 \mathrm{~mJ}$ using a specifically created data base as a function of $\left[k, n, E_{\text {impact }}=14 \mathrm{~mJ}\right]$. In order to enhance the potential of the method and allow its use for a larger range of impact energies, i.e. strain rate, without creating a new database for each specific energy, an interpolation method has been established. It allows filling in the new database without any other FEM simulation.

For example, to obtain a new database with intermediate energies between 8 and $17 \mathrm{~mJ}$ the following equation was used:

$c(x)=\frac{(a(x)-b(x))^{*} E_{\text {impact }}}{(E 1-E 2)}+\frac{\left(E 1^{*} b(x)-E 2^{*} a(x)\right)}{(E 1-E 2)}$

Consider $c(x)$ is the value of radius or load and $E_{\text {impact }}$ is the desired energy, E1 is the greatest energy and $E 2$ is the lowest energy already existing in the database, $a(x)$ and $b(x)$ are the value of radius or load for E1 and E2 respectively.

\section{Blind test}

To check the performances of the inverse identification and to validate databases, blind tests were conducted using virtual materials assumed to be following Hollomon's law. Other virtual materials that do not follow this specific mechanical behaviour were also tested (referred as "non-Hollomon" materials later) to observe the limits of the analysis.

With this technique the reliability of the interpolation method by testing the virtual material with an interpolated database can be also tested.

These blind tests were also used to check the reliability of the interpolation method by testing virtual materials at intermediate energies.

\subsection{Blind test on virtual materials assumed to be following Hollomon's law}

The inverse identification has been first applied on virtual materials assumed to be following Hollomon's law.

Several materials have been tested with different values of $k$ and $n$ at two different energies. The elastic properties have been chosen as $E=210 \mathrm{GPa}$ and $v=0.3$. The parameters $k$ and $n$ of virtual tested materials are (see Fig. 6):

$k$ : $[800 ; 1500 ; 2800 ; 3300] \mathrm{MPa}$

$n:[0.12 ; 0.25 ; 0.35 ; 0.47]$

Two databases were used, i.e. a first database for $E_{\text {impact }}=8 \mathrm{~mJ}$ and another database for $E_{\text {impact }}=11 \mathrm{~mJ}$. The database for $E_{\text {impact }}=11 \mathrm{~mJ}$ was obtained by the interpolation method using two initial databases, the database of $8 \mathrm{~mJ}$ and $17 \mathrm{~mJ}$.

Fig. 7 presents the original virtual material that assumed to be following Hollomon's law with the couple $k=800 \mathrm{MPa}$ and $n=0.12$ and the result obtained by inverse identification i.e. Hollomon's law with the couple $k=972 \mathrm{MPa}$ and $n=0.2$. The relative error between the 2

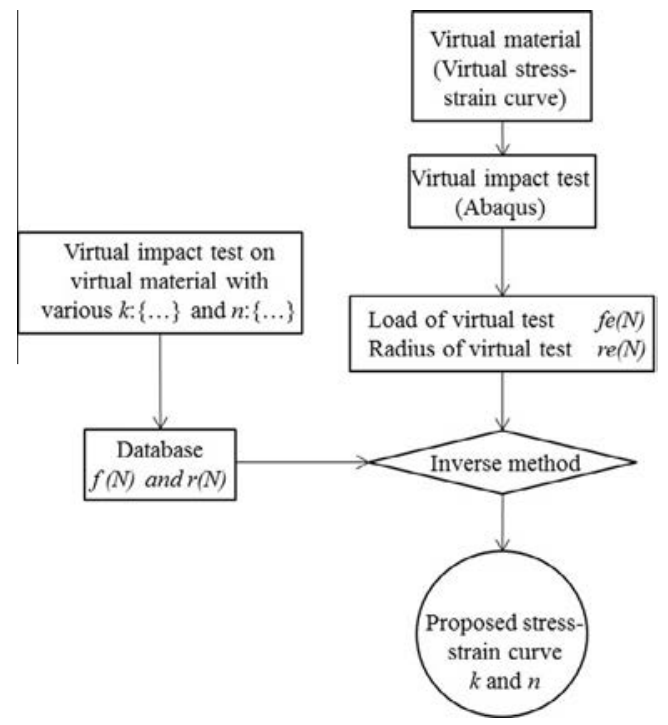

Fig. 6. Flow diagram describe the identification of the stress-strain curve of virtual materials.

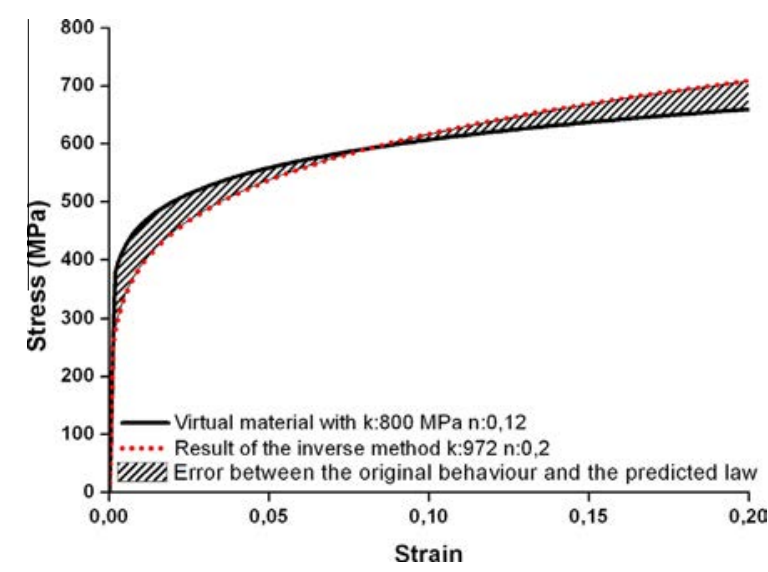

Fig. 7. Error between virtual stress-strain curve which simulates the virtual impact and the predicted stress-strain curve obtained by inverse method, in this case it's around 5\%.

curves was then estimated to $5 \%$ using Eq. (5), with $f_{o}(\varepsilon)$ is the Hollomon equation of the original stress-strain curve and $f_{p}(\varepsilon)$ is the Hollomon equation of the predicted stress-strain curve obtained by the inverse identification.

Fig. 8 shows the error values between the fit of the original stress-strain curve and the fit of the stress-strain curve obtained by the inverse identification in the range of deformation from 0 to 0.2 for both energies. Fig. 8(a) at energy $8 \mathrm{~mJ}$ shows that the error does not exceed $14 \%$ whereas Fig. 8(b) at energy $11 \mathrm{~mJ}$ shows that the error does not exceed $15 \%$.

$E r=100 * \frac{\sqrt{\int_{0}^{0.2}\left(f_{o}(\varepsilon)-f_{p}(\varepsilon)\right)^{2} d \varepsilon}}{\sqrt{\int_{0}^{0.2}\left(f_{o}(\varepsilon)\right)^{2} d \varepsilon}}$

As both cases lead to similar and reduced error values, the validity of the interpolation method can be confirmed. 

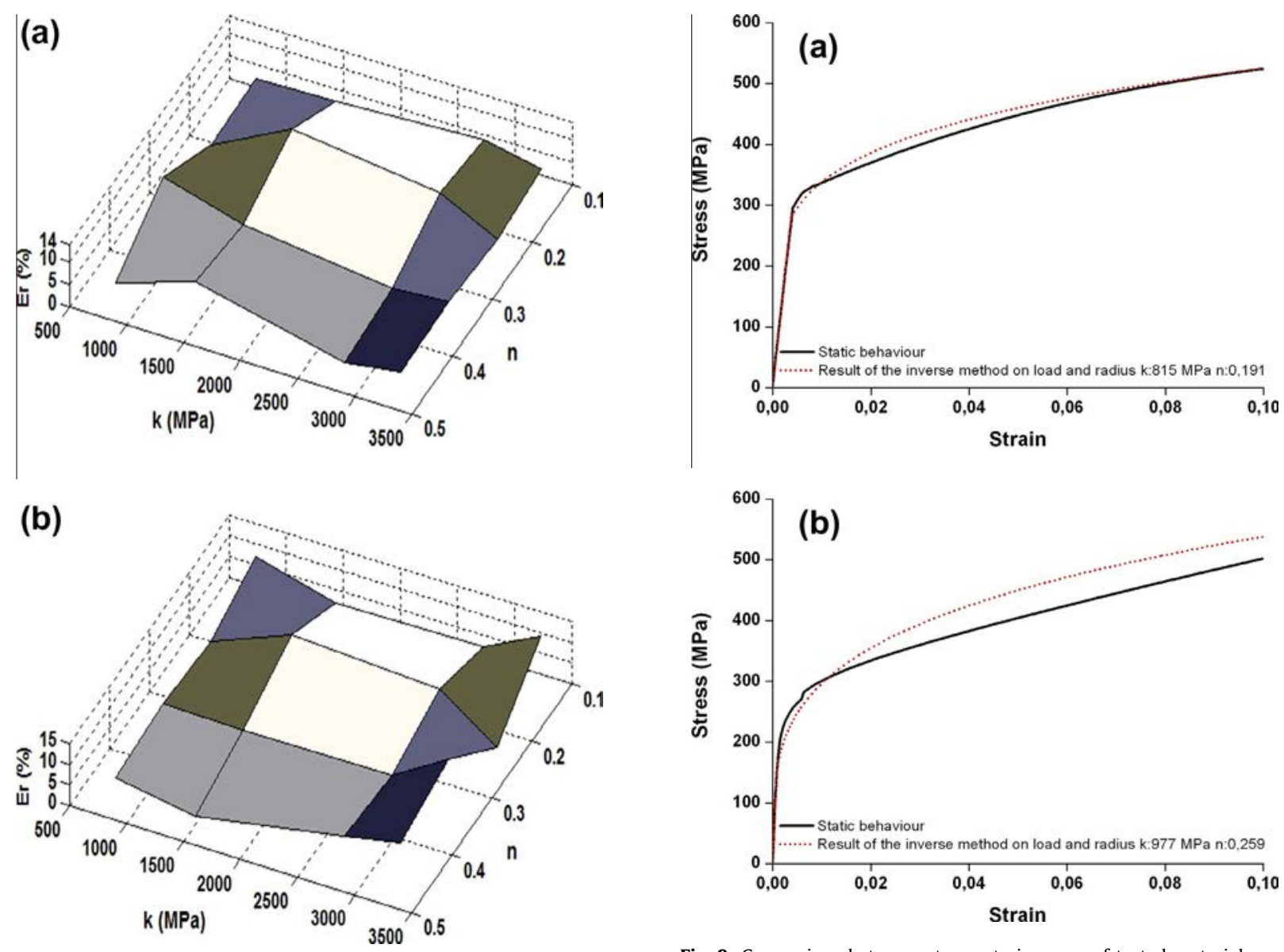

Fig. 8. Relative error Er of (Eq. (4)) as function of $k$ and $n$ : (a); impact energy $8 \mathrm{~mJ}$, (b); impact energy $11 \mathrm{~mJ}$.

The obtained error also shows that the inverse identification allows to propose a good estimation of the stressstrain curve of virtual materials assumed to be following Hollomon's law using only the result of 10 virtual impacts.

\subsection{Blind test on virtual "Non-Hollomon" materials}

"Non-Hollomon" materials were also tested by the inverse identification in order to check the limit of the proposed approach.

Virtual AU4G aluminium alloy and AISI316L were chosen for these blind tests. Static stress-strain curves obtained through traction tests were used as reference curves and for the initial numerical simulations. The results of the virtual impact test obtained by using these stress-strain curves were then used in the inverse method to estimate the Hollomon's laws that can fit the reference curves.

Fig. 9 presents the results of blind tests applied to Aluminium alloy AU4G (Fig. 9(a)) and AISI316L (Fig. 9(b)). The inverse identification is launched with databases created with the specific elastic properties of each material.

Fig. 9. Comparison between stress-strain curve of tested materials and results of inverse method: (a) AU4G; impact energy 17 mJ, (b) AISI316L; impact energy $14 \mathrm{~mJ}$.

In the case of AU4G (Fig. 9a), both initial and proposed stress-strain curves are almost superimposed. For AISI316L (Fig. 9(b)), the proposed stress-strain curve is slightly above the initial ones but remains in the $15 \%$ error bar that has been observed for Hollomon's virtual materials (Fig. 7).

In order to investigate this effect and understand how the accuracy of the inverse identification may be related to the impact energy or material parameters, blind tests have been performed on the same virtual material but at different energies. Fig. 10 shows the obtained results and demonstrate that the agreement between both curves depends on the impact energy. The higher energy, the better approach.

This may be explained by the range of radius and load values generated during the virtual impacts. Using low energies, the induced plastic deformation remains limited leading to small values of strain and stress. As information on high strain region are missing, the inverse identification proposes a less accurate stress-strain curve. Using high impact energies generating larger scar radius and load seems to lead to improved solutions. Depending on the material plastic properties, threshold energy value leading to acceptable solution quality is likely to exist. 

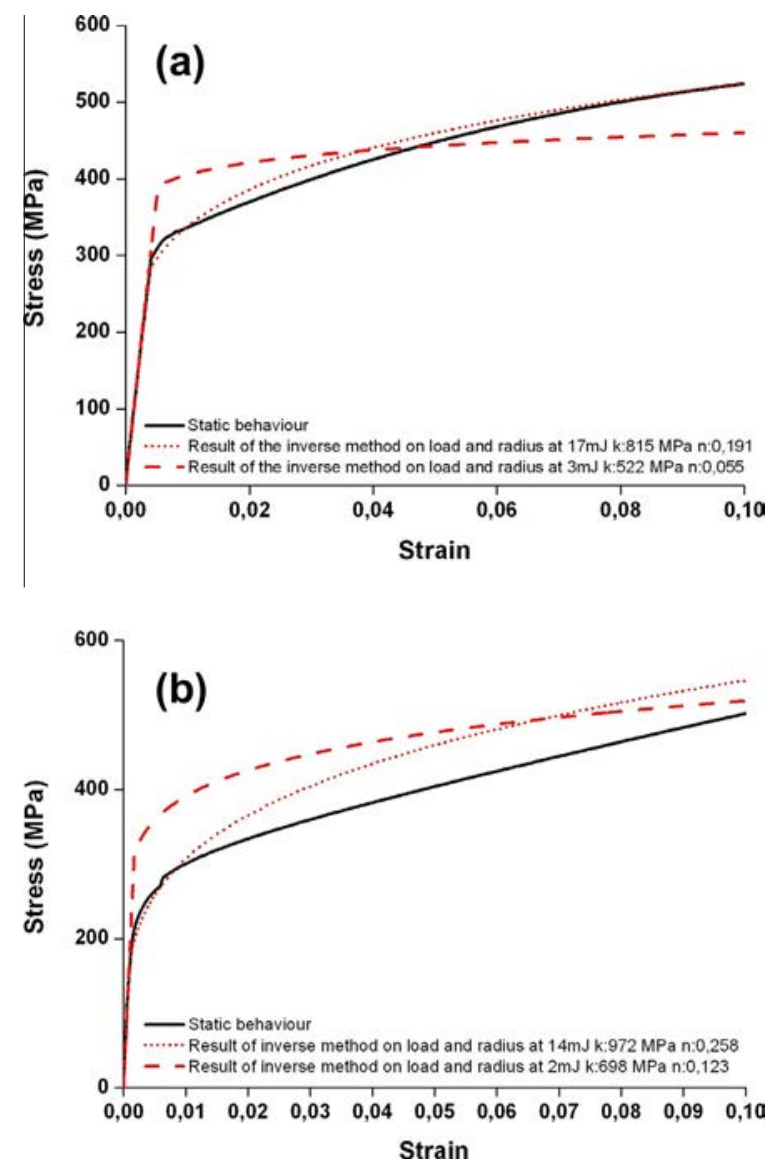

Fig. 10. Comparison between stress-strain curve of virtual material and results of inverse method for various impact energies: (a) AU4G; (b) AISI316L.

According to Fig. 10(a), this energy threshold value seems to be less than $17 \mathrm{~mJ}$ for AU4G whereas energy of $14 \mathrm{~mJ}$ appears to be still insufficient for AISI316L.

It can be obviously deducted from the curves drawn in Figs. 9 and 10 that the proposed inverse method leads to the definition of a Hollomon's law parameters that may also be used as an approach of the stress-strain curve for "non-Hollomon" materials.

Thanks to that approached stress strain curve and FEM models, physical quantities which are difficult and costly to measure by experimental test may be estimated. Fig. 11 shows for example the equivalent plastic strain distribution on the AU4G after 1, 3 and 6 dynamic impacts at $17 \mathrm{~mJ}$. This figure shows that important hardening is localised underneath the indenter. Such information are of main interest in case of shot peening study for example. The proposed stress strain curve should lead to improved residual stress predictions.

\subsection{Comparison studies}

Like all new methods for materials characterisation, comparison studies must be carried out to determine its potential to provide reliable data.
5.3.1. An analytical approach to the stress strain curve

Based on Tabor, Johnson and Hill's work (Hill et al., 1989; Johnson, 1985; Tabor, 2000), Kermouche et al. (2008) presented a direct method to estimate the stressstain curve of materials under sharp indentation test using the mean pressure and the contact radius. The mean pressure can be written as: $P m=L / \pi a^{2}$ where $L$ is the applied load and $a$ is the contact radius. We propose here to extend this model to spherical indentation. It yields:

$\sigma_{r}=\frac{\zeta \frac{a}{R} P m}{\gamma_{p} \zeta \frac{a}{R}-(1-\zeta B) \frac{P m}{\mathrm{E}}}$

and

$\varepsilon_{r}=(1-\zeta B) \frac{\sigma_{r}}{\mathrm{E}}+\zeta \frac{a}{R}$

In the previous expression $\gamma_{p}$ is the ratio between the mean pressure and the yield stress representative value. The value of 2.8 suggested by Tabor (2000) is generally admitted and has been used in this study. The value of $B$ is equal to: $\left(1-v^{2}\right) 3,3 \pi / 4$ and $\zeta$ is given by Eq. (8):

$\zeta=\frac{4}{3 \pi \gamma_{p}\left(1-v^{2}\right)}$

$R$ and the elastic properties of the materials being known, the value of $B$ and $\zeta$ can easily be deduced. Considering experimental values of load and radius, $\mathrm{Pm}$ can be calculated and the stress-strain couple of values for the tested material can be determined. This leads to the analytical determination of a set of points that can be plotted and compared to the virtual and proposed stress-strain curves in order to obtain a certain confidence level on the result of the inverse identification.

\subsection{Comparison with stress-strain curves obtained by previous methods}

The above presented results have also to be compared to former ones using depth and radius values instead of load and radius (Kermouche et al., 2013).

Fig. 12 shows different stress-strain curves obtained for two different virtual materials using an inverse identification launched on radius and load values and another inverse identification launched on the depth and radius values (Kermouche et al., 2013). Points issued from the analytical approach (Kermouche et al., 2008) are also plotted.

Comparing the stress-strain curves obtained using the inverse methods, the inverse identification launched on load and radius values provides better results than the inverse identification launched on the radius and depth values. Moreover a good agreement may be observed between the analytically determined points and the proposed stress-strain curves especially for medium strain values [0.04-0.08]. Similarly to the previously results, analytical results obtained at low stress-strain values appear less accurate whereas those obtained for higher stressstrain couples better fit the proposed stress-strain curves. 


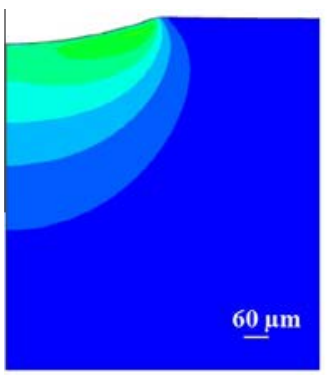

One impact

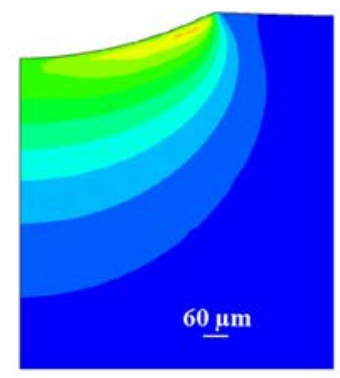

3 impacts

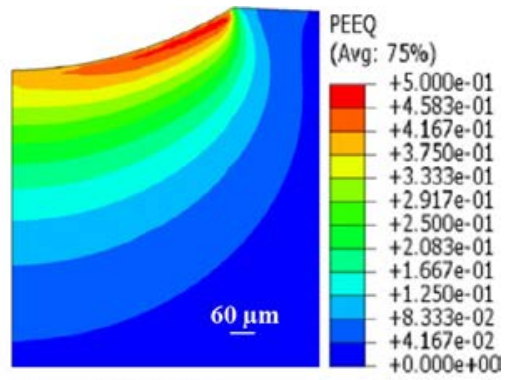

6 impacts

Fig. 11. Equivalent strain rate contour plots on $A U 4 G$ for 1,3 and 6 impacts for an impact energy of $17 \mathrm{~mJ}$.
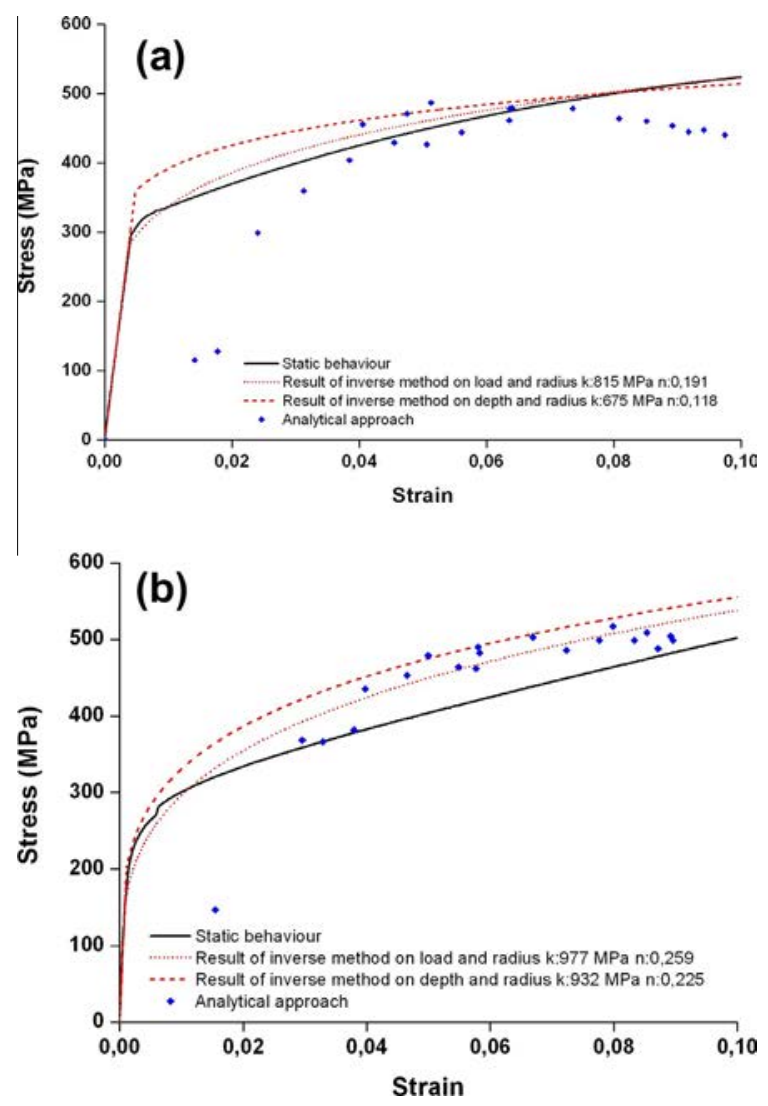

Fig. 12. Comparison between stress-strain curves obtained by inverse method performed over several data and the analytical approach for: (a) AU4G; impact energy $17 \mathrm{~mJ}$, (b) AISI316L; impact energy $14 \mathrm{~mJ}$.

\section{Application on real material}

After the validation of the proposed inverse method on virtual materials (exhibiting hollomon or non hollomon mechanical behaviour), the same study has been applied to real materials. For the first application, a real but model metallic substrate having isotropic and fine grains structure has been selected in order to avoid any uncontrolled microstructural effects. All samples were mirror polished prior to any impact test.

According to these criteria, commercially pure copper has been chosen for the validation of the inverse method.
Moreover, the question of the temperature rise during the impact, may be specifically addressed for these copper samples. Proposed by Johnson (1985), Eq. (9) enables to estimate the possible thermal effects during impacts

$\Gamma=\frac{T}{3 Y_{d} R^{3}}$

Where $T$ is the impact energy, $Y_{d}$ is the dynamic yield stress and $R$ is the indenter radius.

According to Johnson (1985) a value of $\Gamma$ between 10 and $10^{-1}$ indicates that temperature effects are negligible. In the case of copper, $\Gamma$ is always under than $10^{-1}$ (with $Y_{d} \approx 350 \mathrm{MPa}$ ).

Fig. 13 shows the typical FCC fine grain structure leading to a global isotropic behaviour. As the main objective of the present study is to validate the inverse identification, no specific correlation between the microstructure of this material and the obtained strain-stress curve has been made. All tests were performed using a $2 \mathrm{~mm}$ diameter Zirconia ball of and the impact energy was fixed at $11 \mathrm{~mJ}$. The $11 \mathrm{~mJ}$ database used in the inverse identification was created by the interpolation technique between two fully computed databases at $9 \mathrm{~mJ}$ and $17.2 \mathrm{~mJ}$. Fig. 14 compares the results of the inverse identification on load and radius developed in the present study, the results of the previously method on depth and radius (Kermouche et al., 2013) and those of the analytical approach.

Fig. 14 clearly shows that the inverse method applied on radius and load is able to propose a better approach. In particular the proposed stress-strain curve is in good

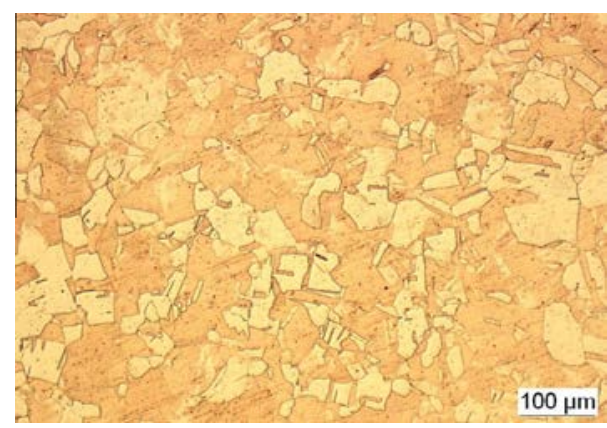

Fig. 13. Grain structure of the commercially pure copper. 


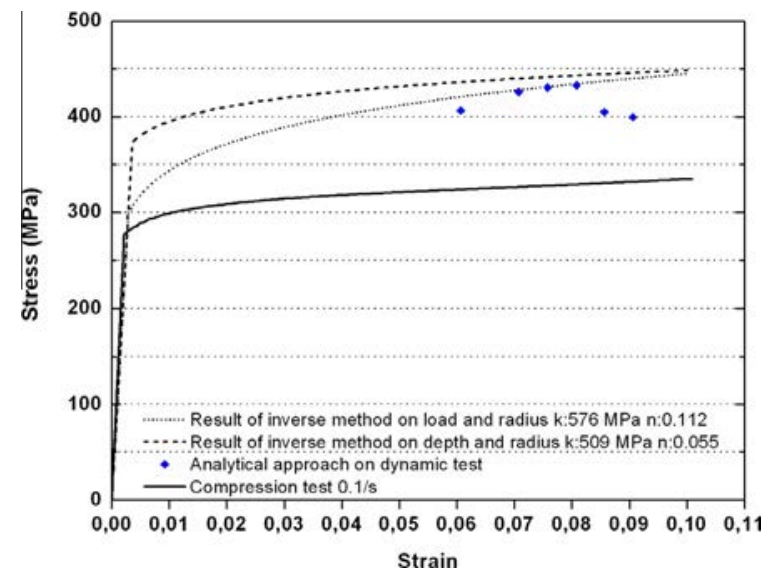

Fig. 14. Results of the inverse methods and analytical approach on the commercially pure copper.

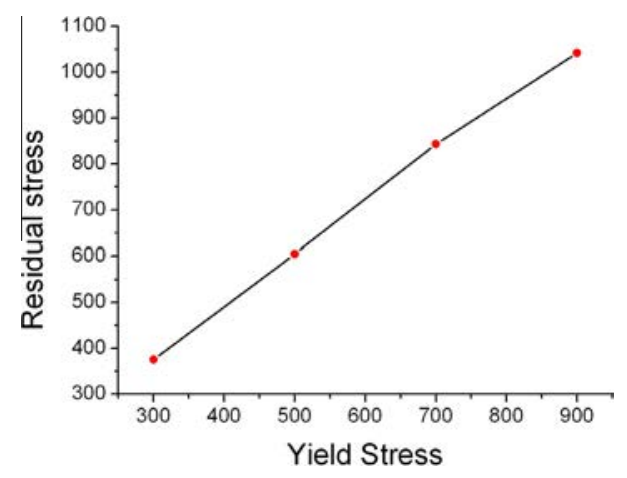

Fig. 15. Evolution of residual stress versus yield stress.

agreement with the results obtained from the analytical approach.

These tests performed on commercially pure copper substrates show clear evidence of the potential development of the new method. The proposed inverse method allows to determine stress-strain curve only using 10 repeated impacts performed at controlled energy.

\section{Conclusion}

This paper proposes a new method based on the coupling of numerical and experimental impact tests. Using the results of radius and load obtained after a micro-impact test at given energy, an inverse identification method has been developed. Based on the results of numerical simulations performed using different parameters i.e. Hollomon stress-strain curve, an approach of the stress-stain curve of material under dynamic compression conditions may be obtained.

Firstly, virtual materials were tested. The results show that the inverse method using a Hollomon stress-strain curve databases is able to propose an approach similar to that of the material stress-strain curve.

Moreover, it also appears that the inverse identification using the radius and load results is more accurate than that using the radius and depth data (Kermouche et al., 2013).
Depending on the expected behaviour of the material (Hollomon model or not), the proposed stress-strain curve may be more or less accurate.

Even if this newly developed method does not allow identifying the exact behaviour of materials at high strain rates unlike tests where the load and displacement are continuously measured during the test, it allows giving an approach similar to that of the stress-strain curve of materials within a $15 \%$ error range (estimation on virtual materials). Furthermore, the main advantage of this technique is that it does not require a large volume of materials no specific testing samples and it's proved to be not time consuming once the database is created.

Future works on real materials exhibiting different microstructures and mechanical behaviours are now planned to establish the inverse method limits and recommendations in terms of energy or impacting ball size.

\section{Acknowledgements}

The authors would like to express their deepest gratitude to the University of Technology of Belfort-Montbéliard (UTBM) and Pays de Montbéliard Agglomération (PMA) for their financial support. They also would like to thank Th. Couturier et D. Schlegel from the UTBM-IMSI Dept. for their technical help.

\section{Appendix A}

Relationship between yield stress and compressive residual stress

The objective of the shot peening surface treatment is to create a compressive residual stress field to improve the fatigue resistance of engineering parts. Here we propose to highlight the strong relation between the impact-induced compressive residual stress level and the yield stress of materials. For that purpose, simulations have been carried out using the FE model developed in this paper using an elastic-perfectly plastic material $(E=210000 \mathrm{MPa}$, $\mathrm{Nu}=0.3, Y=\{300 ; 500 ; 700 ; 900\} \mathrm{MPa}, \mathrm{Ec}=17 \mathrm{~mJ})$, where $E$ is the Young modulus, $Y$ is the Yield stress and Ec is the kinetic energy. Results are plotted in Fig. 15. It appears that the maximum compressive residual stress is almost linear with the yield stress in this case. It thus points out the need to identify the stress-strain curves of the materials inside the shot peening affected zone and at a strain rate close representative of this kind of impact-based treatment to predict the effects of such a process. The method presented in this paper can be used in that way

\section{References}

Abramov, V.O., Abramov, O.V., Sommer, F., Gradov, O.M., Smirnov, O.M., 1998. Surface hardening of metals by ultrasonically accelerated small metal balls. Ultrasonics 36, 1013-1019. http://dx.doi.org/10.1016/ S0041-624X(98)00027-4.

Beghini, M., Bertini, L., Fontanari, V., 2006. Evaluation of the stress-strain curve of metallic materials by spherical indentation. Int. J. Solids Struct. 43, 2441-2459. http://dx.doi.org/10.1016/j.ijsolstr. 2005.06.068. 
Chollacoop, N., Dao, M., Suresh, S., 2003. Depth-sensing instrumented indentation with dual sharp indenters. Acta Mater. 51, 3713-3729. http://dx.doi.org/10.1016/S1359-6454(03)00186-1.

Collin, J.-M., Mauvoisin, G., Bartier, O., El Abdi, R., Pilvin, P., 2009 Experimental evaluation of the stress-strain curve by continuous indentation using different indenter shapes. Mater. Sci. Eng. A 501 140-145. http://dx.doi.org/10.1016/j.msea.2008.09.081.

Collin, J.-M., Mauvoisin, G., Pilvin, P., 2010. Materials characterization by instrumented indentation using two different approaches. Mater. Des. 31, 636-640. http://dx.doi.org/10.1016/j.matdes.2009.05.043.

Collin, J.-M., Mauvoisin, G., Pilvin, P., El Abdi, R., 2008. Use of spherical indentation data changes to materials characterization based on a new multiple cyclic loading protocol. Mater. Sci. Eng. A 488, 608-622. http://dx.doi.org/10.1016/j.msea.2008.01.041.

Dao, M., Chollacoop, K., Van Vliet, K.J., Venkatesh, T.A., Suresh, S., 2001 Computational modeling of the forward and reverse problems in instrumented sharp indentation. Acta Mater., 3899-3918

Hill, R., Storakers, B., Zdunek, A.B., 1989. A theoretical study of the Brinell Hardness test. Proc. R. Soc. Lond. Math. Phys. Sci. 423, 301-330. http://dx.doi.org/10.1098/rspa.1989.0056.

Huang, Y., Liu, X., Zhou, Y., Ma, Z., Lu, C., 2011. Mathematical analysis on the uniqueness of reverse algorithm for measuring elastic-plastic properties by sharp indentation. J. Mater. Sci. Technol. 27, 577-584. http://dx.doi.org/10.1016/S1005-0302(11)60111-4.

Huber, N., Tsakmakis, C., 1999. Determination of constitutive properties from spherical indentation data using neural networks. Part ii: plasticity with nonlinear isotropic and kinematichardening. J. Mech. Phys. Solids 47, 1589-1607. http://dx.doi.org/10.1016/S00225096(98)00110-0.

Jaspers, S.P.F.C., Dautzenberg, J.H., 2002. Material behaviour in conditions similar to metal cutting: flow stress in the primary shear zone. J. Mater. Process. Technol. 122, 322-330.

Johnson, K.L., 1985. Contact Mechanics. Cambridge University Press.

Kermouche, G., Grange, F., Langlade, C., 2013. Local identification of the stress-strain curves of metals at a high strain rate using repeated micro-impact testing. Mater. Sci. Eng. A 569, 71-77. http://dx.doi.org/ 10.1016/j.msea.2013.01.020.

Kermouche, G., Loubet, J.L., Bergheau, J.M., 2008. Extraction of stressstrain curves of elastic-viscoplastic solids using conical/pyramidal indentation testing with application to polymers. Mech. Mater. 40 271-283. http://dx.doi.org/10.1016/j.mechmat.2007.08.003.

Lamri, S., Langlade, C., Kermouche, G., 2013. Damage phenomena of thin hard coatings submitted to repeated impacts: Influence of the substrate and film properties. Mater. Sci. Eng. A 560, 296-305. http://dx.doi.org/10.1016/j.msea.2012.09.070.

Lamri, S., Langlade, C., Kermouche, G., Martinez, V., 2010. Estimation of the stress relief induced in $\mathrm{CrN}$ thin films by buckling. Mater. Sci. Eng. A 527, 7912-7919. http://dx.doi.org/10.1016/j.msea.2010.08.067.

Lee, J., Lee, C., Kim, B., 2009. Reverse analysis of nano-indentation using different representative strains and residual indentation profiles. Mater. Des. 30, 3395-3404. http://dx.doi.org/10.1016/j.matdes.2009. 03.030 .

Lu, J., Suresh, S., Ravichandran, G., 2003. Dynamic indentation for determining the strain rate sensitivity of metals. J. Mech. Phys. Solids 51, 1923-1938. http://dx.doi.org/10.1016/j.jmps.2003.09.007.

Miao, H.Y., Larose, S., Perron, C., Lévesque, M., 2011. Numerical simulation of the stress peen forming process and experimental validation. Adv. Eng. Softw. 42, 963-975. http://dx.doi.org/10.1016/j.advengsoft. 2011.05.025.

Moussa, C., Bartier, O., Mauvoisin, G., Hernot, X., Collin, J.-M., Delattre, G., 2014a. Experimental and numerical investigation on carbonitrided steel characterization with spherical indentation. Surface and Coatings Technology 258 (2014), 782-789. http://dx.doi.org/ 10.1016/j.surfcoat.2014.07.080.

Moussa, C., Hernot, X., Bartier, O., Delattre, G., Mauvoisin, G., 2014b. Identification of the hardening law of materials with spherical indentation using the average representative strain for severa penetration depths. Mater. Sci. Eng. A 606, 409-416. http:/ dx.doi.org/10.1016/j.msea.2014.03.123.

Sekkal, A.C., Langlade, C., Vannes, A.B., 2005. Tribologically transformed structure of titanium alloy (TiAI6V4) in surface fatigue induced by repeated impacts. Mater. Sci. Eng. A 393, 140-146.

Subhash, G., Koeppel, B.J., Chandra, A., 1999. Dynamic indentation hardness and rate sensitivity in metals. J. Eng. Mater. Techs. Trans. ASME 121, 257-263.

Sundararajan, G., Tirupataiah, Y., 2006. The localization of plastic flow under dynamic indentation conditions : I. Experimental results. Acta Mater. 54, 565-575.

Systems, D., 2011. Abaqus Explicit.

Tabor, D., 2000. The hardness of metals. Clarendon Press; Oxford University Press, Oxford; New York.

Tirupataiah, Y., Sundararajan, G., 1991. A dynamic indentation technique for the characterization of the hight strain rate plastic flow behaviour of ductile metals and alloys. J. Mech. Solids 39, 243-271. 